\title{
Channeling the power of scorpion venom
}

Scorpions, cockroaches and clawed frogs may sound like ingredients in an ancient recipe for witches' brew. But bringing these animals together in a series of experiments has uncovered a new understanding of a more mundane problem: pesticide resistance in insects. The results of this recent study may help scientists to develop better pesticides-no spell book required.

Scorpions produce a variety of toxins that target different channels and receptors in their prey's neuromuscular systems. Common targets of these toxins are the voltage-gated sodium channels, proteins involved in rapid electrical signaling in nerve and muscle cells. Most organisms have a broad array of sodium channel variants with different specific properties, and some scorpion toxins selectively affect certain types of channels. For example, certain toxins are effective in insects but not in mammals. As a result, some of these toxins are valuable in the creation of insecticides. Unfortunately, over time, insects become resistant to toxins that target sodium channels. A research group led by Ke Dong (Michigan State University, East Lansing)

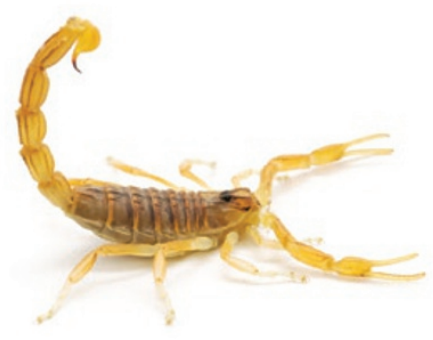

desert scorpion, Leiurus quinquestriatus hebraeus. Next, they engineered oocytes from clawed frogs (Xenopus) to express sodium channels from German cockroaches (Blattella germanica). They then investigated the interaction between the toxin and the sodium channel (J. Biol. Chem. 286, 15781-15788; 2011).

They identified one sodium channel variant that was extremely sensitive to the scorpion toxin. Comparing it with other less-sensitive variants, they found several amino acid changes in the channel's protein sequence that had affected its sensitivity to scorpion toxin. One of these changes, in the voltage-sensing module of domain III of the pore-forming $\alpha$-subunit, was responsible for the hypersensitivity of this channel variant to scorpion toxin. Additionally, they found that changes to other specific amino acids in the channel's protein sequence increased the channel's toxin sensitivity. The results elucidate the mechanism underlying sodium channel sensitivity to scorpion toxin, information that could be exploited to produce more effective pesticides.

\section{Monica Harrington}

\section{UNRAVELING SLEEP}

According to a recent study, localized regions of a rat's brain can enter into an 'off' state that resembles sleep, even when the rat appears to be completely awake (Nature 472, 443-447; 2011). Single cortical neurons may even enter into this sleep state while the rat is awake. These findings suggest that the electrical activity of single cortical neurons is the basic unit of sleep. Further studies on this topic may help researchers better understand functional deficits in sleep-derived people.

Neuroimaging studies have found that sleep deprivation is associated with changes in blood flow and metabolism in the brain (J. Sleep Res. 9, 207-231; 2000). However, the underlying neuronal activity changes in response to sleep deprivation are poorly understood. In an effort to better understand neuronal effects of the prolonged awake state, Guilio Tononi of the University of Wisconsin-Madison and colleagues studied neuronal activity in rats. They implanted microwire arrays in the deep layers of the frontal motor and parietal cortices in adult rats. The team then recorded local neuronal activity when the rats were in spontaneous sleep and awake states. To measure global neuronal activity, the team used electroencephalography.

When the rats were awake, local neuronal activity was, as expected, generally characterized by low-amplitude fast waves and theta waves. In contrast, when the rats were asleep, high-amplitude slow waves dominated and the neurons fluctuated between 'on' and 'off' states. The research team then kept the rats awake for up to 4 hours longer than normal. Single units in both the frontal and parietal brain regions of the sleep-deprived rats sporadically entered into the off state. These periods of 'local sleep' became more frequent as the rats were kept awake for longer periods.

The researchers then tested whether the off state in the motor cortex of sleep-deprived rats would affect the ability of rats to reach for a food reward. They found that poorer performance in this task was associated with increased occurrence of the off state in the motor cortex.

The authors note these findings "raise the intriguing possibility that 'local sleep' in an awake brain may be responsible for cognitive impairments due to sleep deprivation or restriction." They note, however, that further study is needed to more precisely associate specific performance failures with the occurrence of off periods in specific neuronal subsets. Further study could also help determine whether local sleep in awake rats is adaptive or maladaptive. 